\title{
Induction of BCL2-Interacting Killer, BIK, is Mediated for Anti-Cancer Activity of Curcumin in Human Head and Neck Squamous Cell Carcinoma Cells
}

\author{
Yue Xi1, 2, Hang Gao1, Michael U. Callaghan², Andrew M. Fribley2,3, Danielle M. Garshott², Zhi-Xiang Xu1,4, \\ Qinghua Zeng ${ }^{1,4,}$ and $\mathrm{Yu}$-Lin $\mathrm{Li}^{1,}{ }^{1}$ \\ 1. The Key Laboratory of Pathobiology, Ministry of Education, Norman Bethune College of Medicine, Jilin University, Changchun 130021, \\ China \\ 2. Carmen and Ann Adams Department of Pediatrics, Department of Otolaryngology, Wayne State University, Detroit, MI 48201, USA \\ 3. Molecular Therapeutics Program, Karmanos Cancer Institute, Detroit, MI 48201, USA \\ 4. Division of Hematology/Oncology, Department of Medicine, University of Alabama at Birmingham, Birmingham, AL, 35294, USA
}

$\bowtie$ Corresponding authors: Qinghua Zeng: zengnwu@yahoo.com or Yu-lin Li: ylli@jlu.edu.cn

(C) 2015 Ivyspring International Publisher. Reproduction is permitted for personal, noncommercial use, provided that the article is in whole, unmodified, and properly cited. See http://ivyspring.com/terms for terms and conditions.

Received: 2014.11.26; Accepted: 2015.01.03; Published: 2015.02.12

\begin{abstract}
Naturally occurring diarylheptanoid curcumin (CUR), a principal component of the Asian spice turmeric, has been shown to have anti-cancer effects in many tumor types. However, a detailed mechanism regarding CUR induced tumor cell killing remain to be comprehensively explored. Using two head neck squamous cell carcinoma (HNSCC) cell lines FaDu (hypopharyngeal) and Cal27 (tongue), we demonstrated a novel mechanism by which CUR levies the cytotoxic effect. We found that CUR induced upregulation of pro-apoptotic Bik, down-regulation of survival signaling by AKT and NF-KB prior to the induction of the caspase-cascade reduction of cell proliferation, are primary mechanisms of CUR-induced cell death, thus providing insights into the anti-tumor activity of CUR in HNSCC cells.
\end{abstract}

Key words: Curcumin, Bik, caspase-9, caspase-3, apoptosis, squamous cell carcinoma, natural product, head and neck cancer

\section{Introduction}

Head and neck squamous cell carcinoma (HNSCC) is one of major causes of cancer morbidity and mortality in the United States and across the world despite concerted efforts to development of new therapeutic approaches $[1,2]$. Many challenges exist in the management of HNSCC due to both the location of the tumor and the impairment of essential functions, such as speech and swallowing, caused by efforts to relieve tumor burden [3]. Conventional therapeutic strategies, which include surgery, chemotherapy, and radiation, are found to only be effective in $50 \%$ of the cases [4] and do not significantly improve survival rate over past several decades. Platinum-based compounds, such as cisplatin, are the cornerstone of treatment for these cancers. Cisplatin exerts a dose-dependent response where higher doses induce apoptosis in a larger fraction of tumor cells [5]; cisplatin-resistance a major contributor to morbidity [6]. There is a tremendous need to develop new tumor specific and molecularly targeted agents to alleviate this challenge [7]. Single agent targeted therapies, such as monoclonal antibodies, that inhibit a variety of oncogenic kinases or growth factor pathways have been extensively pursued in recent years [8], but they have not led to significant increases in durable response nor increased survival. Signal transduction in cancer cells is a sophisticated process that involves receptor tyrosine kinases (RTKs) that eventually trig- 
ger multiple cytoplasmic kinases, which are often serine/threonine kinases [9]. Clinical-trial data has indicated that targeting only one of the kinases involved in these signaling pathways results in limited or sporadic responses [10]. Recent evidence suggests that the simultaneous inhibition of multiple kinase-driven signal pathways may facilitate optimization of the overall therapeutic benefit [11]. It is urgent to identify a single agent with the ability to target multiple biomarkers [12-14].

Recent studies have shown that diferuloylmethane, or curcumin (CUR), that is an active component of the perennial herb Curcuma longa (turmeric), possesses potent anti-inflammatory, antioxidant and a broad spectrum of tumor suppressive activities [15]. CUR has been shown to suppress multiple cell signaling pathways, as well as inhibit cell proliferation, invasion, metastasis and angiogenesis [15]. It has been reported that CUR suppresses activation of nuclear factor-kappa B (NF- $\kappa \mathrm{B})$ and signal transducer and activator for transcription 3 (STAT-3) [16], while at the same time down-regulates the expression of genes such as B-cell lymphoma (Bcl)-2, cyclooxygenase-2 (Cox2), matrix metalloproteinase-9 (MMP-9), G1/S-specific cyclin D1 and cell adhesion molecules $[17,18]$.

Although numerous studies have demonstrated the chemopreventative potential of CUR against a wide variety of tumors, HNSCC focused studies are limited. This report sheds new light on the mechanistic process by which CUR suppressed the growth of HNSCC cells via the down-regulation of cell survival signaling pathways and enhanced Bcl-2-interacting killer (Bik)-mediated programmed cell death.

\section{Material \& Methods}

\section{Cell lines, reagents and proliferation assays}

The hypopharyngeal carcinoma cell line FaDu and the tongue squamous cell carcinoma cell line Cal27 were purchased from ATCC (Manassas, VA). Cells were maintained in ATCC-formulated Eagle's Minimum Essential Medium and supplemented with $10 \%$ fetal bovine serum. CUR was purchased from Sigma-Aldrich (Saint Louis, MO). Cell viability was measured using Cell Counting Kit-8 (Dojindo Laboratories, Tokyo) according to the manufacturer's protocol and incubation at $37^{\circ} \mathrm{C}$ for 2 hours. Absorbance at $450 \mathrm{~nm}$ was measured using an EL-808 96-well plate reader (BioTek). All cell survival assays were performed at least three times with triplicate samples in 96-well plates using 10,000 cells/well. Error bars represent the standard deviation of a representative experiment. Statistical significance between the means of each cell line was calculated using a two-tailed
Student's $t$-test. A $p$ value $<0.05$ was considered statistically significant.

\section{Clonogenic survival assay}

One thousand of $\mathrm{FaDu}$ or $\mathrm{Cal} 27$ cells were seeded in six-well plates and treated with CUR or DMSO control, as indicated. After one hour, the medium was replaced with completed (drug-free) medium and the cultures were allowed to grow for 10 12 days. The colonies were fixed and stained with $0.5 \%$ crystal violet in absolute ethanol, photographed and counted using a dissection microscope.

\section{Immunoblotting and DNA Fragmentation}

$\mathrm{FaDu}$ and Cal27 whole cell lysates were prepared for immunoblot analysis as previously described [19]. $50 \mu \mathrm{g}$ of protein lysate was resolved on 8 - 12\% SDS-polyacrylamide gels and transferred to PVDF membranes (BioRad, CA). Membranes were probed overnight at $4^{\circ} \mathrm{C}$ with monoclonal or polyclonal antibodies. Antibodies were purchased from the following sources: pro-apoptosis Bcl-2 family antibody sampler kit, pro-survival Bcl-2 family antibody sampler kit, caspase-9, cleaved caspase-3, PARP, phospho-EGFR (Try1068), phospho-HER3/ErbB3 (Tyr1222), phospho-AKT (Ser473), phospho-p44/42 MAPK(Erk1/2), phospho-STAT3 (Ser727), phospho-STAT3 (Tyr705), phospho-NF-кB p65 (Ser536) from Cell Signaling Technology. SuperSignal west pico ECL-HRP substrate (Thermo Scientific) was used for protein detection. To demonstrate uniform loading of samples, each represented membrane was stripped and re-probed with monoclonal antibody; EGFR (F4), ErbB-3 (RTJ.2), AKT (B-1), Erk1/2 (MK1), cyclin D1 (A-12), cyclin D2 (B-6) or $\beta$-actin (Santa Cruz Biotechnology). Purified genomic DNA was isolated as previously described [20]. To verify equal protein loading, after the membrane was stripped with Stripping Buffer, the membrane was re-probed with anti- $\beta$-actin $(1: 10,000)$ monoclonal antibodies. To examine DNA fragmentation, cells were lysed, and the soluble fraction was extracted with phenol-chloroform (Fisher Scientific). Fragmented DNA was separated on a 1.5\% agarose gel as described previously [21].

\section{Results}

\section{CUR suppresses HNSCC proliferation and colony formation}

Concentration response assays were performed with FaDu and Cal27 cells for 24 hours to evaluate the effect of CUR on HNSCC cell growth. CCK-8 cell survival assays revealed that CUR significantly inhibited the viability of FaDu and Cal27 cells (Figure 1A). 12.5 $\mu \mathrm{M}$ CUR (the approximate $\mathrm{IC}_{50}$ at 24 hours), contin- 
ued to reduce proliferation over a 72-hour period (Figure 1B). To further study the anti-proliferative effect observed in FaDu and Cal27, colony forming assay was performed to determine if CUR was cytotoxic or cytostatic. CUR exposure resulted significant inhibition of colony formation (Figure 1C and D). These data indicate that at low concentrations short term exposure of CUR can inhibit HNSCC cell growth and that it becomes increasingly toxic over a very narrow range.

\section{CUR induced caspase activation in FaDu cells}

We next sought to determine the extent to which apoptosis might govern the anti-proliferative effect of
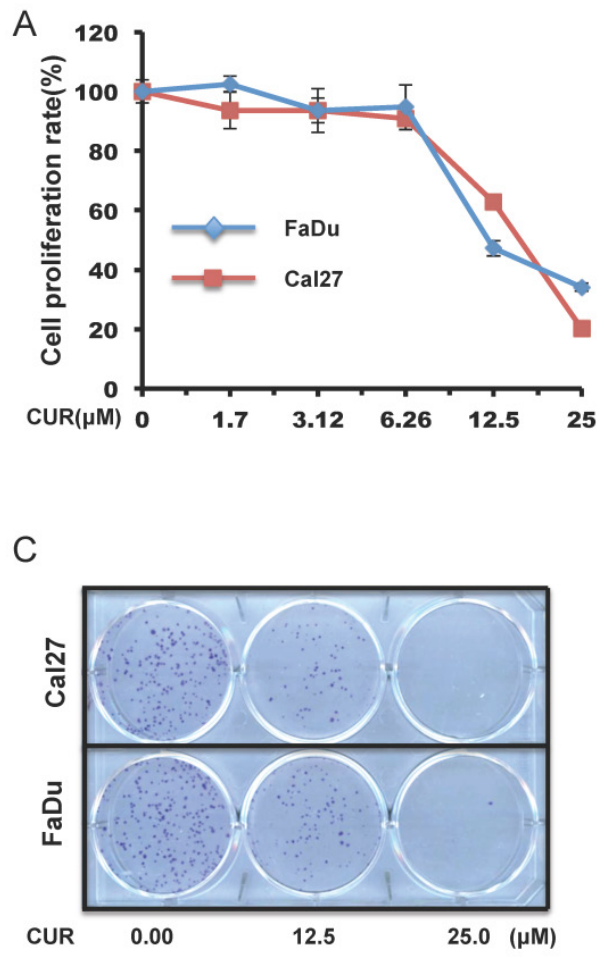

CUR. Electrophoretically resolved genomic DNA harvested from $\mathrm{FaDu}$ cells exposed to increasing concentrations of CUR for 24 hours revealed fragmentation consistent with apoptosis (Fig. 2A). To determine the kinetics of CUR-induced apoptosis Western immunoblot analysis was performed with whole cell lysates harvested from FaDu cells at 24 and 48 hours. After 24 and 48 hours, $12.5 \mu \mathrm{M}$ CUR induced bands consistent with the active forms of PARP and caspases-9 and -3 in the cells (Figure 2B). Considered together, these results suggest that CUR induced apoptosis in $\mathrm{FaDu}$ cells by activating the intrinsic mitochondrial-mediated apoptotic pathway.

$\mathrm{B}$
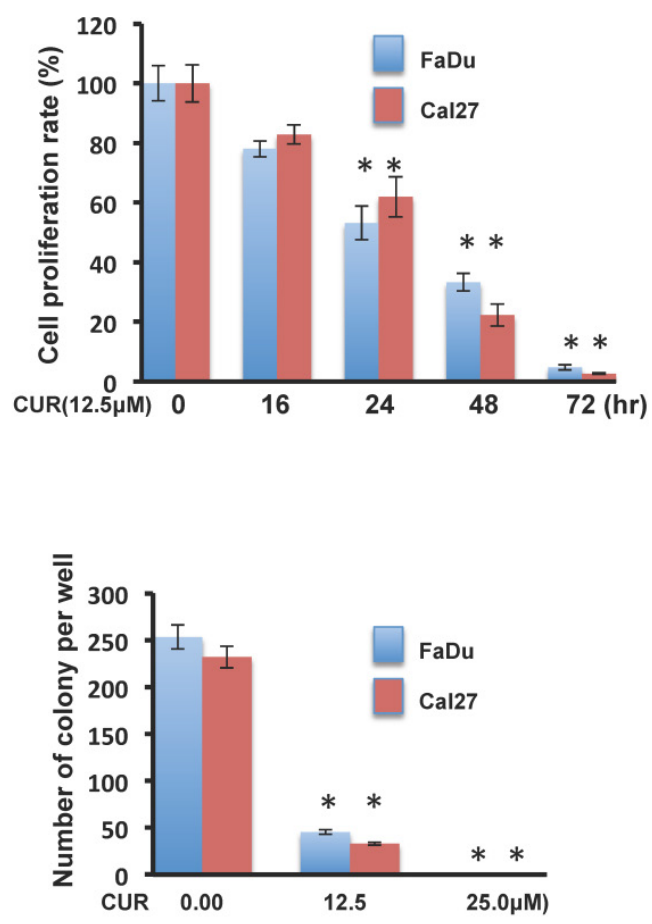

Figure 1. CUR inhibited proliferation and colony formation in HNSCC cell lines. A, B. Color metric cell viability CCK-8 assays were performed in FaDu and Cal27 cell lines after exposure to a serial dose-response of CUR for 24 hours, and after exposure to $12.5 \mu M$ CUR for 16, 24, 48 and 72 hours (two-tailed Student's t-test, $p<0.05$ ). C, D. Clonogenic cell survival assays in FaDu and Cal27 cells was treated for 1 hour with 12.5 and $25 \mu$ M of CUR (two-tailed Student's $t$-test, $\mathrm{p}<0.05)$.

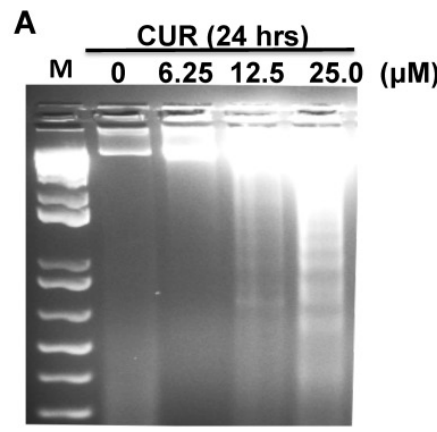

FaDu



FaDu

Figure 2. CUR induced apoptosis in HNSCC cells. A. Genomic DNA electrophoresis was performed to observe apoptotic DNA fragmentation in FaDu cells following 24 hours exposure to varying doses of CUR. B. Immunoblot analysis of whole-cell lysates from FaDu cells treated with CUR and probed with monoclonal antibodies for PARP, pro-caspase-9 and cleaved caspase-3. For immunoblot analysis, each membrane was stripped and re-probed with monoclonal $\beta$-actin. " 0 " indicates equal molar DMSO control. 


\section{Bcl-2 family pro-apoptotic Bik and Bim pro- teins were induced by CUR in FaDu cells}

The Bcl-2 family of proteins plays a critical role in the regulation of cytochrome $c$ release from the mitochondria during intrinsic apoptosis. To determine whether CUR induced the apoptotic cascade through regulation of $\mathrm{Bcl}-2$ family members immunoblot analysis was performed with whole cell lysates from FaDu cells treated for 24 and 48 hours. The results indicated that while the expression levels of anti-apoptotic proteins $\mathrm{Bcl}-\mathrm{xL}, \mathrm{Bcl}-\mathrm{w}, \mathrm{Bcl}-2$, and Mcl-1 were not significantly influenced (Fig. $3 \mathrm{~A}$ ), the pro-apoptotic protein Bik and Bim were significantly increased (Fig. 3 B). It has been reported that up-regulation of Bik activates Bax/Bak which in turn inhibits the function of $\mathrm{Bcl}-2 / \mathrm{Bcl}-\mathrm{xL}$, thus resulting in cytochrome $\mathrm{C}$ release and ultimately triggering caspase activation [22]. Collectively, these results suggested that CUR might regulate activation of the caspase-casade through induction of Bik and Bim.

\section{CUR inhibited cell growth and survival signal- ing networks in HNSCC}

Accumulated data has revealed that CUR targets key cellular signaling pathways including NF- $\mathrm{B}$, PI3K/AKT, MAPK/Erk and JAK/STAT[23, 24]. We have reported that up-regulation of PI3K/AKT and MAPK/Erk signaling pathways are associated with HNSCC progression [21, 25, 26]. The effects of 12.5 $\mu \mathrm{M}$ CUR on constitutive PI3K/AKT and MAPK/Erk activity in HNSCC cells was examined following 24 hours exposure. Immunoblot analysis revealed decreased expression of phospho-AKT and phospho-Erk1/2 in both Cal27 and FaDu (Fig. 4 A). Since both PI3K/AKT and MAPK/Erk signaling pathways require EGFR activation for regulation of cell proliferation and survival, expression of phospho-EGFR and phospho-HER3/ErbB3 was assessed. CUR led to a slight reduction in phospho-EGFR and phospho-HER3/ErbB3 (Figure 4 B). To determine whether CUR modulates NF-KB and STAT-3 activation phosphorylation of NF-kB and STAT-3 in both FaDu and Cal27 cells was measured. CUR reduced phosphorylation of NF-kB and STAT-3 in both cells lines compared with their controls (Fig. 4 C). Having established that CUR could reduce the activity of these key survival pathways, we further examined whether CUR could affect modulators of cell proliferation. We found that CUR considerably inhibited expression of cyclin D1 and cyclin D2 in Cal27 cells, while only a moderate reduction in expression was observed in the FaDu cells (Fig. 4 D). Considered together, these data suggest that the collective inhibition of survival signaling networks is an important feature of CUR-mediated anti-proliferation in HNSCC.



Figure 3. CUR induced Bcl-2 family pro-apoptotic Bik and Bim protein expression in HNSCC. FaDu cells were treated in the presence or absence of $12.5 \mu M$ CUR for 24, and 48hrs. Immunoblot analysis of whole-cell lysates from FaDu cells treated with CUR and probed with antibodies for $\mathrm{Mcl}-1$, $\mathrm{Bcl}-\mathrm{x}_{\mathrm{L}}, \mathrm{Bcl}-2, \mathrm{Bak}, \mathrm{Bax}, \mathrm{Bik}$, and Bim. Each membrane was stripped and re-probed with monoclonal $\beta$-actin. " 0 " indicates equal molar DMSO control.

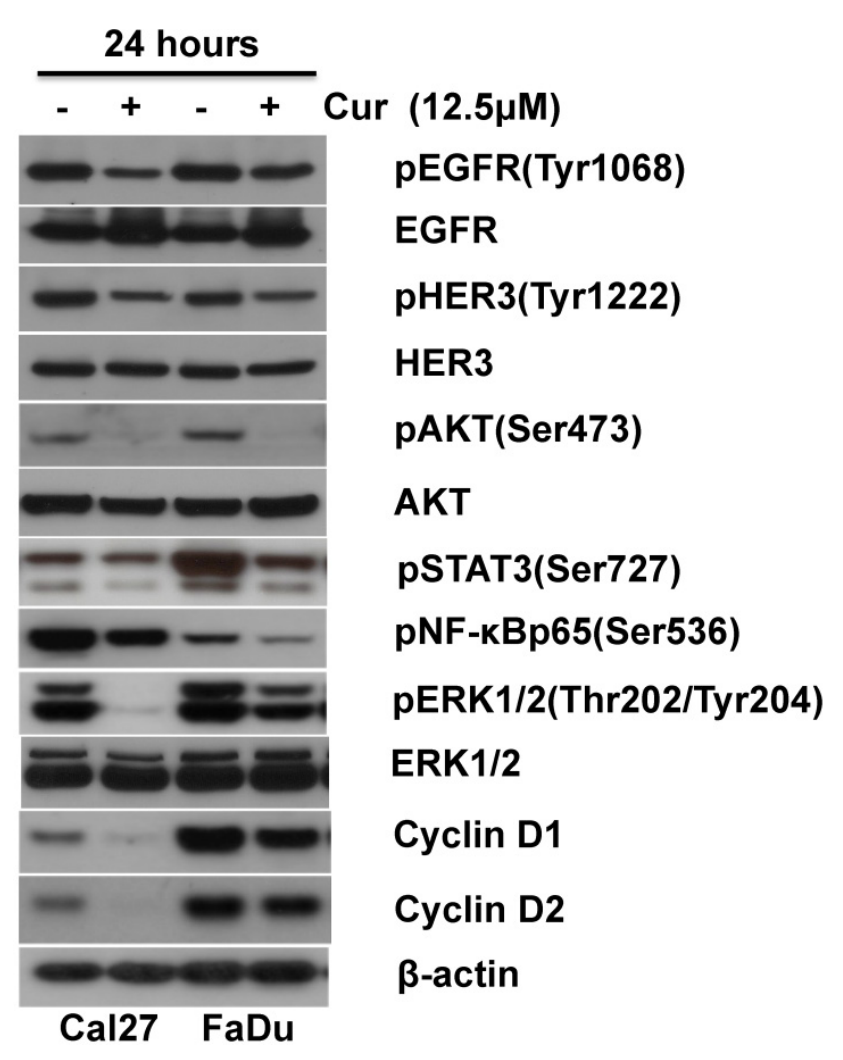

Figure 4. CUR inhibited pro-survival signaling pathways in HNSCC cells. Immunoblot analysis of whole-cell lysates from $\mathrm{FaDu}$ and $\mathrm{Cal} 27$ cells exposed to CUR with antibodies for phospho-HER3/ErbB3, phospho-EGFR, phospho-STAT3, phospho-NF-KBp65, phospho-AKT, phospho-ERK1/2, cyclin D1 and cyclin D2. Normal Her3, EGFR, AKT, ERK1/2 and $\beta$-actin were used as loading controls. " - " indicates equal molar DMSO control. 


\section{Discussion}

More than $60 \%$ of FDA-approved anti-cancer drugs are natural products or derived from them [27, 28]. Natural products that exhibit the ability to suppress activation of cell survival pathways, while selectively enhancing cell death in malignant cell populations are generally regarded to have the greatest therapeutic potential. CUR is ideal in this regard and it is abundant and inexpensive [15]. One of the major challenges limiting the clinical utility of CUR is sub-therapeutic bioavailability. Recently though, there have been several very promising reports that the CUR analogs UBS109 and H-4073 (with improved pharmacokinetics) inhibit growth and enhance cisplatin-mediated killing respectively, in murine HNSCC xenograft models $[29,30]$. Continued interest in moving CUR toward the clinic, with studies such as these, underscores the necessity to more completely elucidate the subcellular pathways and processes modulated by CUR. The current study demonstrates, for the first time, that CUR inhibits cell proliferation and induces apoptosis in HNSCC FaDu and Cal27 cells subsequent to increased protein expression of pro-apoptotic Bcl-2 family members Bik and Bim, and the suppression of several key cell survival signaling pathways.

Programmed cell death is a normal physiological response necessary to maintain homeostasis during tissue development. Coding mutations in many genes such as TP53, FAT1, CDKN2A, NOTCH1, CASP8, are common in HNSCC and can facilitate evasion of apoptosis and drive tumorigenesis and resistance to chemotherapy [31]. The Bcl-2 family is comprised of both pro- and anti-apoptotic members classified by their Bcl-2 homology domains (BH1 - BH4) [32]. Pro-apoptotic members can contain $\mathrm{BH} 1, \mathrm{BH} 2$ and $\mathrm{BH} 3$ domains (Bax, and Bak) or just the BH3 domain, such as Bim, Bik, PUMA and NOXA [32, 33]. Anti-apoptotic members such as Bcl-2, Bcl- $\mathrm{x}_{\mathrm{L}}, \mathrm{Mcl}-1$, $\mathrm{Bcl}-\mathrm{w}$ contain the $\mathrm{BH} 1, \mathrm{BH} 2, \mathrm{BH} 3$ and $\mathrm{BH} 4$ domains [32]. Activation of intrinsic apoptotosis leads to Bax and Bak homo-oligomerization and permeabilization of the mitochondrial membrane which facilitates the release of cytochrome $\mathrm{c}$ and precipitates assembly of the apoptosome [32]. Bik is the founding member of the BH3-only family proteins [34], and a number of reports have established that ectopic overexpression of Bik results in apoptotic cell death [20, 35, 36]. Our finding that Bik and Bim protein levels are high while HNSCC are undergoing apoptosis suggests that pro-apoptotic signaling by the Bcl-2 family might be an important feature of CUR-induced cell death. The simultaneous cleavage and activation of PARP, Caspase 9 and Caspase 3 further support this notion.

Numerous in vitro and in vivo models have demonstrated that the anti-cancer potential of CUR depends on its ability to modulate NF- $\kappa B$, AKT, MAPK, p53, Nrf2, Notch-1, JAK/STAT, $\beta$-catenin, AMPK and other signal transduction pathways [23]. STAT3 is an oncogene that is overexpressed in approximately $82 \%$ of HNSCC cases [37-39]. Activation of STAT3 leads to proliferation and survival mediated by the induction of specific target genes, such as cyclin D1, Bcl-2, and Bcl- $x_{\mathrm{L}}[37,40]$. Interruption of STAT3 impedes cancer cell growth and enhances apoptosis in HNSCC [41, 42]. Constitutive NF-кB signaling protects HNSCC and other cancer cells from apoptotic cell death through the regulation of genes involved in inflammation, proliferation, metastasis, tumor cell adhesion [43-45]. The current study demonstrates that CUR markedly decreases the phosphorylation of STAT3 and NF- $\mathrm{kB}$ in HNSCC FaDu and Cal27 cells. The cytotoxic effect of CUR in HNSCC is preceded by the complementary inhibition of survival signals that prevent apoptosis and the induction of the pro-apoptotic Bcl-2 family members Bike and Bim.

In HNSCC, STAT3 activation has been implicated as the principal link to aberrant autocrine/paracrine transforming growth factor (TGF) a/epidermal growth factor receptor (EGFR) signaling [46] and other upstream events, such as interleukin-6/Janus kinase (JAK) signaling [47]. EGFR is over-expressed in a wide variety of solid tumors, including $80-90 \%$ of HNSCC [48]. Monoclonal antibodies, and low-molecular weight tyrosine kinase inhibitors (TKI) specifically directed against these receptors have been utilized in the clinic, however monotherapy with EGFR inhibitors has seen with only limited success, with response rates between 5 $15 \%$ in recurrent and/or metastatic cancers $[49,50]$. Herein, our findings demonstrate that CUR induced inhibition of PI3K/Akt, MAPK/Erk, STAT3 and the up-stream mediators EGFR1 and HerbB3 in both HNSCC FaDu and Cal27 cells.

In conclusion, present study indicated that CUR is a potential anticancer drug of natural product. Our results suggested that CUR suppressed the growth of HNSCC FaDu cells via activation pro-apoptotic Bik resulted in induction of apoptosis in the cells through inhibition of pro-survival signaling pathways.

\section{Supplementary Material}

Supplemental Figure 1.

http://www.jcancer.org/v06p0327s1.pdf

\section{Acknowledgements}

These studies were supported by pilot funds from the UAB Breast Cancer NCI SPORE project and NIH grant CA 108741. Portions of this work were also 
supported by the National Natural Science Foundation of China grants \#81272243 and \#81271853. Portions of these studies were supported by NIH grants R00 DE019678 and R03 MH089787, the Children's Hospital of Michigan Foundation and the Wayne State University Fund for Medical Research (AMF).

\section{Competing Interests}

The authors have declared that no competing interest exists.

\section{References}

1. Jemal A, Siegel R, Ward E, Murray T, Xu J, Thun MJ. Cancer statistics, 2007. CA: a cancer journal for clinicians. 2007; 57: 43-66

2. Parkin DM, Bray F, Ferlay J, Pisani P. Global cancer statistics, 2002. CA: a cancer journal for clinicians. 2005; 55: 74-108.

3. Sen B, Saigal B, Parikh N, Gallick G, Johnson FM. Sustained Src inhibition results in signal transducer and activator of transcription 3 (STAT3) activation and cancer cell survival via altered Janus-activated kinase-STAT3 binding. Cancer research. 2009; 69: 1958-65.

4. Boehm AL, Sen M, Seethala R, Gooding WE, Freilino M, Wong SM, et al. Combined targeting of epidermal growth factor receptor, signal transducer and activator of transcription-3, and Bcl-X(L) enhances antitumor effects in squamous cell carcinoma of the head and neck. Molecular pharmacology. 2008; 73: 1632-42.

5. Sorenson CM, Eastman A. Mechanism of cis-diamminedichloroplatinum(II)induced cytotoxicity: role of G2 arrest and DNA double-strand breaks. Cancer research. 1988; 48: 4484-8.

6. Matta A, Ralhan R. Overview of current and future biologically based targeted therapies in head and neck squamous cell carcinoma. Head \& neck oncology. 2009; 1 : 6.

7. Bernier J, Bentzen SM, Vermorken JB. Molecular therapy in head and neck oncology. Nature reviews Clinical oncology. 2009; 6: 266-77.

8. Knowles LM, Stabile LP, Egloff AM, Rothstein ME, Thomas SM, Gubish CT, et al. HGF and c-Met participate in paracrine tumorigenic pathways in head and neck squamous cell cancer. Clinical cancer research : an official journal of the American Association for Cancer Research. 2009; 15: 3740-50

9. Amit I, Wides R, Yarden Y. Evolvable signaling networks of receptor tyrosine kinases: relevance of robustness to malignancy and to cancer therapy. Molecular systems biology. 2007; 3: 151

10. Faivre S, Djelloul S, Raymond E. New paradigms in anticancer therapy: targeting multiple signaling pathways with kinase inhibitors. Seminars in oncology. 2006; 33: 407-20.

11. Zhang YJ, Tian XQ, Sun DF, Zhao SL, Xiong H, Fang JY. Combined inhibition of MEK and mTOR signaling inhibits initiation and progression of colorectal cancer. Cancer investigation. 2009; 27: 273-85.

12. Adjei AA. Targeting multiple signal transduction pathways in lung cancer. Clinical lung cancer. 2005; 7 Suppl 1: S39-44.

13. Adjei AA, Hidalgo M. Treating cancer by blocking cell signals. Journal of clinical oncology : official journal of the American Society of Clinical Oncology. 2005; 23: 5279-80.

14. Adjei AA, Hidalgo M. Intracellular signal transduction pathway proteins as targets for cancer therapy. Journal of clinical oncology : official journal of the American Society of Clinical Oncology. 2005; 23: 5386-403.

15. Ravindran J, Prasad S, Aggarwal BB. Curcumin and cancer cells: how many ways can curry kill tumor cells selectively? The AAPS journal. 2009; 11: 495-510.

16. Aggarwal BB, Shishodia S. Molecular targets of dietary agents for prevention and therapy of cancer. Biochemical pharmacology. 2006; 71: 1397-421.

17. Zhang F, Altorki NK, Mestre JR, Subbaramaiah K, Dannenberg AJ. Curcumin inhibits cyclooxygenase-2 transcription in bile acid- and phorbol ester-treated human gastrointestinal epithelial cells. Carcinogenesis. 1999; 20: 445-51.

18. Chen H, Zhang ZS, Zhang YL, Zhou DY. Curcumin inhibits cell proliferation by interfering with the cell cycle and inducing apoptosis in colon carcinoma cells. Anticancer research. 1999; 19:3675-80.

19. Fribley A, Zeng Q, Wang CY. Proteasome inhibitor PS-341 induces apoptosis through induction of endoplasmic reticulum stress-reactive oxygen species in head and neck squamous cell carcinoma cells. Molecular and cellular biology. 2004; 24: 9695-704.

20. Fribley AM, Miller JR, Brownell AL, Garshott DM, Zeng Q, Reist TE, et al. Celastrol induces unfolded protein response-dependent cell death in head and neck cancer. Experimental cell research. 2014.

21. Zeng Q, Chen S, You Z, Yang F, Carey TE, Saims D, et al. Hepatocyte growth factor inhibits anoikis in head and neck squamous cell carcinoma cells by activation of ERK and Akt signaling independent of NFkappa B. The Journal of biological chemistry. 2002; 277: 25203-8.
22. Bossy-Wetzel E, Green DR. Caspases induce cytochrome c release from mitochondria by activating cytosolic factors. The Journal of biological chemistry. 1999; 274: 17484-90.

23. Shehzad A, Lee YS. Molecular mechanisms of curcumin action: signal transduction. BioFactors. 2013; 39: 27-36.

24. Wilken R, Veena MS, Wang MB, Srivatsan ES. Curcumin: A review of anti-cancer properties and therapeutic activity in head and neck squamous cell carcinoma. Molecular cancer. 2011; 10: 12.

25. Zeng Q, McCauley LK, Wang CY. Hepatocyte growth factor inhibits anoikis by induction of activator protein 1-dependent cyclooxygenase-2. Implication in head and neck squamous cell carcinoma progression. The Journal of biological chemistry. 2002; 277: 50137-42

26. Zeng Q, Li S, Chepeha DB, Giordano TJ, Li J, Zhang H, et al. Crosstalk between tumor and endothelial cells promotes tumor angiogenesis by MAPK activation of Notch signaling. Cancer cell. 2005; 8: 13-23.

27. Gupta SC, Kim JH, Prasad S, Aggarwal BB. Regulation of survival, proliferation, invasion, angiogenesis, and metastasis of tumor cells through modulation of inflammatory pathways by nutraceuticals. Cancer metastasis reviews. 2010; 29: 405-34.

28. Newman DJ, Cragg GM, Snader KM. Natural products as sources of new drugs over the period 1981-2002. Journal of natural products. 2003; 66: 1022-37.

29. Zhu S, Moore TW, Morii N, Howard RB, Arrendale RF, Reddy P, et al. Synthetic curcumin analog UBS109 inhibits the growth of head and neck squamous cell carcinoma xenografts. Current cancer drug targets. 2014; 14: 380-93.

30. Kumar B, Yadav A, Hideg K, Kuppusamy P, Teknos TN, Kumar P. A novel curcumin analog (H-4073) enhances the therapeutic efficacy of cisplatin treatment in head and neck cancer. PLoS One. 2014; 9; e93208.

31. Martin D, Abba MC, Molinolo AA, Vitale-Cross L, Wang Z, Zaida M, et al. The head and neck cancer cell oncogenome: A platform for the development of precision molecular therapies. Oncotarget. 2014

32. Danial NN, Korsmeyer SJ. Cell death: critical control points. Cell. 2004; 116: 205-19.

33. Tait SW, Green DR. Mitochondria and cell death: outer membrane permeabilization and beyond. Nature reviews Molecular cell biology. 2010; 11: 621-32.

34. Boyd JM, Gallo GJ, Elangovan B, Houghton AB, Malstrom S, Avery BJ, et al. Bik, a novel death-inducing protein shares a distinct sequence motif with Bcl-2 family proteins and interacts with viral and cellular survival-promoting proteins. Oncogene. 1995; 11: 1921-8.

35. Rashmi R, Pillai SG, Vijayalingam S, Ryerse J, Chinnadurai G. BH3-only protein BIK induces caspase-independent cell death with autophagic features in Bcl-2 null cells. Oncogene. 2008; 27: 1366-75.

36. Fribley AM, Miller JR, Reist TE, Callaghan MU, Kaufman RJ. Large-scale analysis of UPR-mediated apoptosis in human cells. Methods in enzymology. 2011; 491: 57-71.

37. Nikitakis NG, Scheper MA, Papanikolaou VS, Sauk JJ. The oncogenic effects of constitutive Stat3 signaling in salivary gland cancer cells are mediated by survivin and modulated by the NSAID sulindac. Oral surgery, oral medicine, oral pathology, oral radiology, and endodontics. 2009; 107: 826-36.

38. Liu B, Ren Z, Shi Y, Guan C, Pan Z, Zong Z. Activation of signal transducers and activators of transcription 3 and overexpression of its target gene CyclinD1 in laryngeal carcinomas. The Laryngoscope. 2008; 118: 1976-80.

39. Nagpal JK, Mishra R, Das BR. Activation of Stat-3 as one of the early events in tobacco chewing-mediated oral carcinogenesis. Cancer. 2002; 94: 2393-400.

40. Song JI, Grandis JR. STAT signaling in head and neck cancer. Oncogene. 2000; 19: 2489-95.

41. Kupferman ME, Jayakumar A, Zhou G, Xie T, Dakak-Yazici Y, Zhao M, et al. Therapeutic suppression of constitutive and inducible JAK \STAT activation in head and neck squamous cell carcinoma. Journal of experimental therapeutics \& oncology. 2009; 8: 117-27.

42. Leeman-Neill RJ, Wheeler SE, Singh SV, Thomas SM, Seethala RR, Neill DB, et al. Guggulsterone enhances head and neck cancer therapies via inhibition of signal transducer and activator of transcription-3. Carcinogenesis. 2009; 30: 1848-56.

43. Prakobwong S, Gupta SC, Kim JH, Sung B, Pinlaor P, Hiraku Y, et al. Curcumin suppresses proliferation and induces apoptosis in human biliary cancer cells through modulation of multiple cell signaling pathways. Carcinogenesis. 2011; 32: 1372-80.

44. Wang CY, Mayo MW, Korneluk RG, Goeddel DV, Baldwin AS, Jr. NF-kappaB antiapoptosis: induction of TRAF1 and TRAF2 and c-IAP1 and c-IAP2 to suppress caspase-8 activation. Science. 1998; 281: 1680-3.

45. Beg AA, Baltimore D. An essential role for NF-kappaB in preventing TNF-alpha-induced cell death. Science. 1996; 274: 782-4.

46. Rubin Grandis J, Zeng Q, Drenning SD. Epidermal growth factor receptor--mediated stat3 signaling blocks apoptosis in head and neck cancer. The Laryngoscope. 2000; 110: $868-74$.

47. Siavash H, Nikitakis NG, Sauk JJ. Abrogation of IL-6-mediated JAK signalling by the cyclopentenone prostaglandin 15d-PGJ(2) in oral squamous carcinoma cells. British journal of cancer. 2004; $91: 1074-80$

48. Dassonville O, Bozec A, Fischel JL, Milano G. EGFR targeting therapies: monoclonal antibodies versus tyrosine kinase inhibitors. Similarities and differences. Critical reviews in oncology/hematology. 2007; 62: 53-61.

49. Cohen EE, Lingen MW, Martin LE, Harris PL, Brannigan BW, Haserlat SM, et al. Response of some head and neck cancers to epidermal growth factor receptor tyrosine kinase inhibitors may be linked to mutation of ERBB2 rather than EGFR. Clinical cancer research : an official journal of the American Association for Cancer Research. 2005; 11: 8105-8.

50. Cooper JB, Cohen EE. Mechanisms of resistance to EGFR inhibitors in head and neck cancer. Head \& neck. 2009; 31: 1086-94. 